\title{
A NEW VIEWPOINT ON THE STRUCTURE OF THE CONSIDERATION SET AND ITS CHANGE
}

\author{
Naoki Akamatsu, Keio University, Japan
}

\begin{abstract}
Many studies have examined the structure of the consideration set; however, few studies have been concentrated on its formation flow or change. Although Jarvis and Wilcox (1973) have stressed the importance of these factors, the actual data show that studies on these aspects of this important topic are seldom performed. The purpose of this study is, first, to provide a new viewpoint on the structure of the consideration set by examining the two factors-"size" and "grade of homogeneity" - through a review of the structure of the consideration set, and by providing data that confirms its usefulness. Next, by using this new approach, the role of the change in the structural patterns of the consideration set is shown. After classifying consumers according to the pattern change, the difference in contact media is compared and considered.
\end{abstract}

The application of the new viewpoint revealed differences in consumers' frequency and the intention of the use of contact media. In addition, significant differences were observed among different structure change patterns in contact media. A discussion and detailed results are described below.

(1) Examining the structure of the consideration set according to "size" and "the grade of homogeneity" is useful, particularly because differences in structure are observed among consumers who experience contact with different media. Next, after consumers are classified according to the pattern change in type of the structure of the consideration set, the average value of the frequency of contact media and the intention of the use of some CGM (Consumer Generated Media) sites are compared between some classified groups. (2) "The consumers for whom neither structure nor brand changes" browse a homepage and a blog site more frequently than "the consumers for whom brands taken into consideration completely differ, though the structure did not change." It is thought that consumers who have loyalty to a specific brand perform information contact positively in order to confirm their selection (c.f. Cherney 2003). (3) The intentions of the use of some CGM sites were higher for "those consumers for whom the grade of homogeneity and size changes" than for "consumers for whom brands taken into consideration completely differ, though structure does not change." As pointed out by Howard and Sheth (1969), this is because the information search is positively related to the stage of the comprehensive decision-making-that is, the former consumers will tend to seek variety. This points out the usefulness of determining the differences in consumer behavior with changes in the structural pattern of the consideration set.

Reference available upon request 